\title{
Association Between Age at Menarche and Metabolic Syndrome in Southwest Iran: A Population-Based Case Control Study
}

\section{Bahman Cheraghian}

Alimentary Tract Research Center, Department of Biostatistics and Epidemiology, School of Public Health, Ahvaz Jundishapur University of Medical Sciences, Ahvaz, Iran.

\section{Nader Saki}

Hearing Research Center, Clinical Sciences Research Institute, Department of Otolaryngology, Head and Neck Surgery, Ahvaz Jundishapur University of Medical Sciences, Ahvaz, Iran.

\section{Zahra Rahimi}

Hearing Research Center, Clinical Sciences Research Institute, Department of Biostatistics and Epidemiology, School of Public Health, Ahvaz Jundishapur University of Medical Sciences, Ahvaz, Iran.

\section{Sara Sarvandian}

Clinical Sciences Research Institute, Department of Biostatistics and Epidemiology, School of Public Health, Ahvaz Jundishapur University of Medical Sciences, Ahvaz, Iran.

Seyed Jalal Hashemi

Alimentary Tract Research Center, Department of Internal Medicine, School of Medicine, Ahvaz Jundishapur University of Medical Sciences, Ahvaz, Iran. Jamileh kaabi

Clinical Sciences Research Institute, Ahvaz Jundishapur University of Medical Sciences, Ahvaz, Iran.

Amal Saki Malehi

Department of Biostatistics and Epidemiology, School of Public Health, Ahvaz Jundishapur University of Medical Sciences, Ahvaz, Iran.

\section{Arman Shahriari}

Alimentary Tract Research Center, Department of Internal Medicine, School of Medicine, Ahvaz Jundishapur University of Medical Sciences, Ahvaz, Iran.

Nahal Nasehi ( $\sim$ Nahalnasehi@yahoo.com )

Fertility, Infertility and Perinatology Research Center, Department of Obstetrics and Gynecology, School of Medicine, Ahvaz Jundishapur University of Medical Sciences, Ahvaz, Iran.

\section{Research Article}

Keywords: Age at menarche, Metabolic Syndrome, Hoveyzeh, PERSIAN cohort, Iran

Posted Date: August 24th, 2021

DOI: https://doi.org/10.21203/rs.3.rs-712759/v1

License: () (1) This work is licensed under a Creative Commons Attribution 4.0 International License. Read Full License 


\section{Abstract}

Background: Age at menarche (AAM) affects women's health outcomes and could be a risk factor for some diseases such as Metabolic Syndrome (MetS). We assessed the association between age at menarche and metabolic syndrome components (obesity, hypertension, type 2 diabetes and cardiovascular disease) in women aged 35 to 70 years in Hoveyzeh, southwest Iran.

Methods: This is a case-control study conducted on 5830 women 35 to 70 years in Hoveyzeh cohort study (HCS), a part of the PERSIAN cohort study, between 2016 to 2018. The case group were women with MetS while the controls were women without MetS. Metabolic syndrome is determined based on standard NCEP-ATP III criteria. Demographic, socioeconomic and reproductive history data were gathered face to face by trained interviews. Also, lab, anthropometrics and blood pressure measurements were assayed for participants. Multiple Logistic Regression was used to estimate the association between age at menarche and metabolic syndrome, with adjustment for potential confounding variables.

Results: The overall mean age at menarche was $12.60 \pm 1.76$ years old. Urban and rural women differed in their age at menarche (at age $12.58 \pm 1.71$ and $12.63 \pm 1.83$ years, respectively). Comparison of the four menarche age groups ( $\leq 10,11-12,13-14,15-16$ years) was statistically different showed between age at menarche and MetS. The odds of having metabolic syndrome for groups with menarche age of 13-14 years and 16-15 years, compared to women with a menstrual age $\leq 10$ years, decreased by $21 \%$ and $20 \%$, respectively.

Conclusion: The present study showed the effect of age at menarche on odds of having MetS in women 35-70 years.

\section{Background}

Menarche, defined as the first menstrual period in a woman's life that is a marker of female puberty and the onset of ovarian and other endocrine functions related to reproductive capacity $(1,2)$. In recent decades, the mean age at menarche has decreased $(1,3)$. Early menarche is often defined as menarche $\leq 11$ years old but some investigators base definition on menarche at $\leq 12$ years (4). Many factors including race, genetic, geographical location, climate, lifestyle, socioeconomic status, physical activity and nutrition affect the early or late age at menarche (5-8). Bratke et al proposed a downward trend in the mean age at menarche results from the increase in the prevalence of overweight and obesity (9).

Age at menarche affects women's health outcomes (10) but the results of the studies were inconsistent. Also the average age of menarche (AAM) in many countries has been reported differently. Some studies showed that Age at menarche can be a risk factor for disease (1) as metabolic syndrome (11-14), ovarian cancer (15), blood pressure (16-18), type 2 diabetes (T2D) (19), gestational diabetes mellitus (20) and cardiovascular disease (21). However

, some other studies have shown that there is no statistically significant relationship between menarche age and diabetes (10) and metabolic syndrome (2, 22).

Metabolic syndrome (MetS) consists of hypertension, hyperglycemia, elevated triglycerides, low high-density lipoprotein cholesterol (HDL-C), and abdominal obesity, and is a major predictive variable for CVD (2). Over the past two decades, the prevalence of metabolic syndrome has increased significantly, especially in developing countries (23). The results of a systematic review showed the overall prevalence of MetS $29 \%$ in Iran (24). The findings in Hoveyzeh cohort study demonstrated that the prevalence of MetS was 39.1\% (25). Some factors such as age, weight, menopause, age at menarche, and genetic factors might be risk factors in MetS development (26).Previous studies have shown inconsistent results for the relationship between age at menarche and MetS. Understanding this association can be effective in therapeutic and preventive interventions design and reducing metabolic syndrome and other cardiovascular diseases. Thus, the goal of the present study was to assess the association between age at the onset of menarche and metabolic syndrome and its components in women 35-70 years in HCS.

\section{Methods}

Study design and participants:

This study was an unmatched case-control study. The participants were women aged 35-70 years who entered the enrolment phase of the Hoveyzeh cohort study (HCS). The HCS is a population-based cohort study conducted in Hoveyzeh County. In this study to assess NCDs in the Arab ethnicity in southwest of Iran in 10009 adults (age 35-70 years) that recruited from May 2016 to August 2018(25). The Hoveyzeh cohort study center is one of the sites of the Prospective Epidemiological Research Studies in IrAN (the PERSIAN Cohort Study), including 180000 Iranian adults. All the steps of registration, implementation and quality control of this study have been performed according to the PERSIAN protocol. (27).

The participants were categorized in two groups with MetS as cases $(n=2660)$ and without MetS as controls $(n=3170)$. Then, relationship of presence or absence of MetS with the age at menarche in the two groups were evaluated. Anthropometric measurements, Blood pressure, Triglycerides, HDL, type2 of diabetes mellitus were measured at the first visit .Also participants were asked about their age at the first menstrual.Women who did not have information about menarche age or whose metabolic syndrome criteria were not measured were excluded from this study.Outcome and exposures were measured equally in the two groups. Informed written consent was obtained from the participants on the day of registration.

Assessment of anthropometric measurements and Blood pressure (BP):

Assessment of anthropometric measurements was done while the participants wearing light clothes and no shoes. Height was measured to the nearest 0.5 $\mathrm{cm}$ by a stadiometer (Seca 206), weight was measured to the nearest $1 \mathrm{~kg}$ using a standing scale (Seca 755). Waist, hip, and wrist circumferences (in cm) 
were measured by Seca locked tape meters. BMI was calculated as weight (kg) divided by squared height (m2). BP was measured by Riester sphygmomanometers, twice (10 min interval) on each arm based on PERSIAN cohort protocol (27).

Assessment of biological measurements:

In Hoveyzeh Cohort Study (HCS) was collected $25 \mathrm{cc}$ of fasting blood from each participant. Blood samples were centrifuged (Sigma, Germany) at 3000 rpm for 10 minutes to separate serum. Then, the required serum levels were measured by BT 1500 autoanalyser (Biotecnica Instruments, Italy). A complete blood count (CBC) was done by the hematology autoanalyser (Nihon Kohden 6510-k, Japan). Urine tests (urine pH, specific gravity) and analysis were also performed (27).

Metabolic syndrome criteria:

Based on the National Cholesterol Education Program-Adult Treatment Panel III guidelines (NCEP ATP3) (28) participants with 3 or more of the 5 below criteria diagnosed as MetS. Abdominal obesity (waist circumstance $\geq 102$ in men and $\geq 88$ in women), serum TG $\geq 150 \mathrm{mg} / \mathrm{dL}$, take hypertriglyceridemia medications, $\mathrm{HDL}<40 \mathrm{mg} / \mathrm{dL}$ in men and $<50$ in women, take drug treatment for low HDL-C, BP $\geq 130 / 85$ mmHg, or take hypertension drugs, FBS $\geq 100$ $\mathrm{mg} / \mathrm{dL}$, take hyperglycemia drugs.

\section{Statistical Analysis:}

To describe the variables, mean and standard deviation (SD) for continuous variables and the number and percent for categorical variables were used. The categorical variables were compared by chi-square test. The independent t-test was used to compare continuous variables between the two groups. Multiple logistic regression was used to assess the association between age at menarche and the MetS by controlling confounder factors. The analyses were carried out with SPSS version 23.0. The Statistical significance was declared if the p-value was less than 0.05 .

\section{Results}

In this study, we recruited 5830 women participants of Hoveyzeh Cohort Study. The mean \pm SD age at menarche in our study was $12.60 \pm 1.76$ years old .Mean age at menarche differed in Urban and rural women (at ages $12.58 \pm 1.71$ and $12.63 \pm 1.83$ years, respectively). Based on wealth index, the prevalence of early age of menarche ( $\leq 10$ years) in poorest groups more than richest groups (26.1\% vs. $11.1 \%)$. The overall prevalence of MetS in the enrollment phase in women 35-70 years in Hoveyzeh cohort study was $45.62 \%$ (25). The characteristics of women with or without Mets were reported in Table 1. Participants with MetS were older than without MetS group. Women with MetS had a less than mean age at menarche and HDL level. Controversy women with MetS had a greater mean BMI, height, weight, waist circumference, hip circumference, SBP, DBP, PR, TC, TG, FBS, ALK, AST, ALT levels. The results of the Chi-square test showed that there was a significant relationship between the variables of Wealth index, Use Alcohol, Smoker, Education, Contraceptive use, and metabolic syndrome $(P<0.0001)$. Also the results demonstrated that the difference between mean age of menarche and mean weight, Waist circumference, Hip circumference, Wrist circumference, SBP, DBP, PR, TC, TG, FBS, ALK, AST, ALT, Gravity, Parity in two groups with and without Metabolic syndrome was significant $(P<0.0001)$. (Table1)

Table 1: Characteristics of study population by metabolic syndrome 


\begin{tabular}{|c|c|c|c|c|}
\hline \multicolumn{2}{|l|}{ Variables } & Cases $(n=2660)$ & Controls $(n=3170)$ & P_value \\
\hline \multicolumn{2}{|l|}{ Age, (years) } & $51.12 \pm 9.17$ & $46.28 \pm 8.54$ & $<0.001^{*}$ \\
\hline \multicolumn{2}{|c|}{ Age at menarche, (years) } & $12.51 \pm 1.78$ & $12.67 \pm 1.74$ & $<0.001^{*}$ \\
\hline \multicolumn{2}{|l|}{ Height, (cm) } & $158.97 \pm 5.66$ & $158.77 \pm 5.68$ & $0.18^{*}$ \\
\hline \multicolumn{2}{|l|}{ Weight, (kg) } & $78.87 \pm 13.95$ & $72.00 \pm 15.11$ & $<0.001^{*}$ \\
\hline \multicolumn{2}{|l|}{ BMI, (kg/m2) } & $31.18 \pm 5.31$ & $28.52 \pm 5.60$ & $<0.001^{*}$ \\
\hline \multicolumn{2}{|c|}{ Waist circumference, (cm) } & $105.67 \pm 10.33$ & $97.73 \pm 12.28$ & $<0.001^{*}$ \\
\hline \multicolumn{2}{|c|}{ Hip circumference, (cm) } & $107.90 \pm 10.07$ & $104.89 \pm 10.33$ & $<0.001^{*}$ \\
\hline \multicolumn{2}{|c|}{ Wrist circumference, $(\mathrm{cm})$} & $17.47 \pm 1.34$ & $16.93 \pm 1.29$ & $<0.001^{*}$ \\
\hline \multicolumn{2}{|l|}{ SBP, (mm Hg) } & $116.43 \pm 20.06$ & $106.08 \pm 15.53$ & $<0.001^{*}$ \\
\hline \multicolumn{2}{|l|}{ DBP, (mm Hg) } & $72.59 \pm 11.42$ & $67.82 \pm 10.02$ & $<0.001^{*}$ \\
\hline \multicolumn{2}{|l|}{ PR } & $80.47 \pm 9.66$ & $78.78 \pm 9.27$ & $<0.001^{*}$ \\
\hline \multicolumn{2}{|l|}{ FBS, (mg/dL) } & $130.61 \pm 60.75$ & $96.37 \pm 28.98$ & $<0.001^{*}$ \\
\hline \multicolumn{2}{|l|}{$\mathrm{HDL}-\mathrm{C},(\mathrm{mg} / \mathrm{dL})$} & $48.21 \pm 10.93$ & $57.17 \pm 11.82$ & $<0.001^{*}$ \\
\hline \multicolumn{2}{|l|}{$\mathrm{TG},(\mathrm{mg} / \mathrm{dL})$} & $197.47 \pm 103.05$ & $111.57 \pm 46.97$ & $<0.001^{*}$ \\
\hline \multicolumn{2}{|l|}{$\mathrm{TC},(\mathrm{mg} / \mathrm{dL})$} & $195.74 \pm 43.31$ & $186.93 \pm 37.93$ & $<0.001^{*}$ \\
\hline \multicolumn{2}{|l|}{ AST } & $17.79 \pm 11.32$ & $17.22 \pm 7.71$ & $0.02^{*}$ \\
\hline \multicolumn{2}{|l|}{ ALT } & $19.21 \pm 12.67$ & $16.76 \pm 11.54$ & $<0.001$ \\
\hline \multicolumn{2}{|l|}{ ALK } & $224.87 \pm 65.71$ & $197.64 \pm 63.41$ & $<0.001^{*}$ \\
\hline \multicolumn{2}{|l|}{ Gravity } & $6.64 \pm 3.15$ & $5.70 \pm 2.87$ & $<0.001^{*}$ \\
\hline \multicolumn{2}{|l|}{ Parity } & $6.07 \pm 2.70$ & $5.24 \pm 2.47$ & $<0.001^{*}$ \\
\hline \multirow[t]{5}{*}{ Wealth index } & poorest & $568(21.35)$ & $740(23.34)$ & \multirow[t]{5}{*}{$0.002^{\star \star}$} \\
\hline & poor & $529(19.89)$ & $736(23.21)$ & \\
\hline & moderate & $546(20.53)$ & $587(18.52)$ & \\
\hline & rich & $537(20.19)$ & $586(18.48)$ & \\
\hline & richest & $480(18.04)$ & $521(16.43)$ & \\
\hline \multirow[t]{2}{*}{ Use Alcohol } & Yes & $4(0.15)$ & $2(0.06)$ & \multirow[t]{2}{*}{$0.30^{\star *}$} \\
\hline & No & 2656 (99.85) & 3168 (99.94) & \\
\hline \multirow[t]{2}{*}{ Smoker } & Yes & $228(8.57)$ & $206(6.50)$ & \multirow[t]{2}{*}{$0.003^{* \star}$} \\
\hline & No & $2432(91.43)$ & $2964(93.50)$ & \\
\hline \multirow[t]{5}{*}{ Education } & Illiterate & $2132(80.15)$ & $2324(73.31)$ & $<0.001^{\star \star}$ \\
\hline & Primary school & $333(12.52)$ & $471(14.86)$ & \\
\hline & Secondary school & $73(2.74)$ & $133(4.20)$ & \\
\hline & Diploma & $74(2.79)$ & $124(3.91)$ & \\
\hline & University & $48(1.80)$ & $118(3.72)$ & \\
\hline Use Contraceptive & Yes & $1582(59.47)$ & $1987(62.68)$ & $0.01^{* \star}$ \\
\hline & No & 1078 (40.53) & 1183 (37.32) & \\
\hline
\end{tabular}

*Independent T -test

${ }^{* *}$ Chi-square test 


\section{Abbreviations}

MetS: Metabolic Syndrome,T2D: Type 2 diabetes,TG: Triglyceride,CVD: Cardiovascular Disease,GDM: Gestational Diabetes Mellitus,BP: Blood Pressure,SBP: Systolic Blood Pressure,DBP: Diastolic Blood Pressure,PR: Pulse Rate,HDL-C: High-Density Lipoprotein Cholesterol,ALT: Alanine Transaminase,FBS: Fasting Blood Sugar,TC: Total Cholesterol,AST: Aspartate Transaminase,ALP: Alkaline Phosphatase,BUN: Blood Urea Nitrogen

The frequency of the four menarche age groups ( $\leq 10,11-12,13-14,15-16$ years) were statistically different between the case and the control groups. This were seen for its all components except for Triglyceride (Table 2).

Table 2. Metabolic syndrome components in women 35-70 years old in the Hoveyzeh Cohort Study( HCS)

\begin{tabular}{|c|c|c|c|c|c|c|c|c|c|c|c|c|}
\hline $\begin{array}{l}\text { Category } \\
\text { of age at } \\
\text { menarche }\end{array}$ & $\mathbf{N}$ & $\begin{array}{l}\text { Metabolic } \\
\text { syndrom }\end{array}$ & P_value & $\begin{array}{l}\mathrm{TG} \\
(\mathrm{mg} / \mathrm{dl}) \geq \\
150 \\
\mathrm{mg} / \mathrm{dL}\end{array}$ & P_value & $\begin{array}{l}\mathrm{HDLC} \\
(\mathrm{mg} / \mathrm{dl})<50\end{array}$ & P_value & $\begin{array}{l}\mathrm{BP} \geq 130 / 85 \\
\mathrm{mmHg}\end{array}$ & P_value & $\begin{array}{l}\mathrm{FBS} \geq 100 \\
\mathrm{mg} / \mathrm{dL}\end{array}$ & P_value & $\begin{array}{l}\text { Abdon } \\
\text { circur }\end{array}$ \\
\hline $\begin{array}{l}\leq 10 \\
\text { years }\end{array}$ & 832 & $412(49.5)$ & 0.01 & $309(37.1)$ & 0.58 & $386(46.4)$ & 0.01 & $257(30.9)$ & 0.03 & $386(46.4)$ & 0.004 & $737(81$ \\
\hline $\begin{array}{l}11-12 \\
\text { years }\end{array}$ & 2001 & 939(46.9) & & 794(39.7) & & $864(43.2)$ & & $565(28.2)$ & & $883(44.1)$ & & 1768 ( \\
\hline $\begin{array}{l}13-14 \\
\text { years }\end{array}$ & 2018 & $880(43.6)$ & & 781(38.7) & & $836(41.4)$ & & $517(25.6)$ & & $805(39.9)$ & & 1753 ( \\
\hline $\begin{array}{l}15-16 \\
\text { years }\end{array}$ & 979 & $429(43.8)$ & & $390(39.8)$ & & $382(39)$ & & $275(28.1)$ & & $406(41.5)$ & & 816(8: \\
\hline
\end{tabular}

${ }^{*}$ Chi-square test

Table 3 shows the odds ratio (OR) and 95\% confidence interval (Cl) for MetS according to age at menarche of the women in the four groups ( $\leq 10 y e a r s, 11-12$ years, 13-14 years, 15-16 years), using age at menarche 15-16 years as the reference category. Model 1 shows crude odds ratios of metabolic syndrome for four menarche age groups, so that the odds of metabolic syndrome at menstrual age of $\leq 10$ years $1.25(95 \% \mathrm{Cl}(1.04-1.51)$; $\mathrm{P}=0.015)$ times more than age at menarche 15-16 years. Model 2 is adjusted for demographic variables (age, education) and life-style (smoking, and physical activity). The odds of having MetS in at menstrual age of $\leq 10$ and $11-12$ years were 1.27 (95\% $\mathrm{Cl}(1.04-1.54) ; \mathrm{P}=0.01)$ and $1.20(95 \% \mathrm{Cl}(1.02-1.41)$; $\mathrm{P}=0.02)$ times more than age at menarche 15-16 years, respectively. Model 3 is adjusted for biological measurements (FBS, TG, TCHOL, HDL, SGOT, SGPT and ALK) and DPB, SPB and PR. The odds of having MetS in at menstrual age of $\leq 10$ years was $1.34(95 \% \mathrm{Cl}(1.03-1.74) ; \mathrm{P}=0.02)$ times more than menstrual age $15-16$ years group. Also results in models 4 demonstrated the odds of having MetS in women with age menarche of $\leq 10$ years was 1.30 (95\% $\mathrm{Cl}(1.07-1.58)$; $\mathrm{P}=0.008)$ times more than menstrual age 15-16 years group, after adjustment for reproductive variables (gravidity, parity, gestational diabetes and pregnancy hypertension, use of oral contraceptives, use of hormone replacement therapy) (Table3)

Table 3: Odds Ratios (and 95\% Confidence Intervals) for MetS by Menarcheal Age in Participants

\begin{tabular}{|c|c|c|c|c|c|c|c|c|c|c|c|c|c|c|c|}
\hline \multirow{3}{*}{$\begin{array}{l}\text { Menarche } \\
\text { (Years) }\end{array}$} & \multirow[t]{3}{*}{$\mathrm{N}$} & \multirow[t]{3}{*}{ Case } & \multicolumn{3}{|c|}{ Model 1} & \multicolumn{3}{|c|}{ Model 2} & \multicolumn{3}{|c|}{ Model 3} & \multicolumn{3}{|c|}{ Model 4} & \multirow{3}{*}{$\frac{\mathrm{M}}{\mathrm{Ol}}$} \\
\hline & & & OR & $95 \% \mathrm{Cl}$ & $P$ & OR & $95 \% \mathrm{Cl}$ & $P$ & OR & $95 \% \mathrm{Cl}$ & $P$ & OR & $95 \% \mathrm{Cl}$ & $P$ & \\
\hline & & & & OddsRatio & & & OddsRatio & & & OddsRatio & & & OddsRatio & & \\
\hline$\leq 10$ & 832 & 412 & $1 / 25$ & 1/04-1/51 & $0 / 015$ & $1 / 27$ & $1 / 04-1 / 54$ & 0/01 & $1 / 34$ & 1/03-1/74 & $0 / 02$ & $1 / 30$ & 1/07-1/58 & 0/008 & $1 /$ \\
\hline $11-12$ & 2001 & 939 & $1 / 13$ & $0 / 97-1 / 32$ & $0 / 11$ & $1 / 20$ & 1/02-1/41 & $0 / 02$ & $0 / 95$ & $\begin{array}{l}0 / 76- \\
1 / 194\end{array}$ & $0 / 70$ & $1 / 15$ & 0/98-1/36 & $0 / 07$ & $0 /$ \\
\hline $13-14$ & 2018 & 880 & 0/99 & $0 / 85-1 / 15$ & $0 / 91$ & $1 / 12$ & 0/95-1/32 & $0 / 15$ & $0 / 92$ & $\begin{array}{l}0 / 740- \\
1 / 152\end{array}$ & $0 / 48$ & $1 / 02$ & $0 / 86-1 / 20$ & $0 / 78$ & $0 /$ \\
\hline $15-16$ & 979 & 429 & 1 & - & - & 1 & - & - & 1 & - & - & 1 & - & - & 1 \\
\hline
\end{tabular}

Model 1: Unadjusted. Model 2: Adjusted demographic variable (Age, Education) AND Life-style (smoking, and Physical activity). Model 3: Adjusted biological FBS, measurements (TG, TCHOL, HDL, SGOT, SGPT, ALK) AND DPB, SPB and PR. Model 4: Adjusted Reproductive variable (Gravidity, parity, has gestational diabetes and pregnancy hypertension, use of oral contraceptives and use of hormone replacement therapy). Model 5: Adjusted Model 2, Model 3 and Model 4

\section{Discussion}

Our study demonstrates the mean age at menarche $12.60 \pm 1.76$ years in women participants of Hoveyzeh Cohort Study. Some studies reported the mean menarche age 12.81 years (95\% Cl: 12.56-13.06) in Iranian girls (29), in the United States 12.8 years (30), 13 years in the Netherlands,12.60 years in Turkey, 13.15 years in Dutch girls (31), $13.6 \pm 1.2$ years among Indian women (32) and in China $15.4 \pm 1.9$ years (21). 
One of the reasons for the early age of menarche in some areas is the hot climate mentioned in the Khatoon study (33). Hoveyzeh has a warm climate in southwestern Iran. This might be the reason for lower the mean age of menarche in this region than Iranian women and women live in cold regions.

This study demonstrated mean age at menarche in urban regions slightly lower than in rural areas. This result is almost confirmed by different studies. Rihlat et al reported urban and rural women differed in their age at menarche (at ages 12.7 and 14.5 years, respectively) (34). Ajong et al showed the strength of association of the urban/rural setting with early menarche (35). Another study reported an earlier age at menarche in the urban and suburban dwellers of Northern Ghana region (36).Also, Marván et al showed urban women had statistically significant earlier ages at menarche than rural women (37).

Overnutrition, obesity and overweight in urban girls may be the cause for this difference in menarche age. Inverse Ramalingam et al (38) showed there was no statistical difference between menarche age in urban and rural regions.

In our study, early age menarche was lower than in the richest women. Inversely Marván et al reported in high SES women reached menarche the earliest and Iow SES women the latest. The historical decline in age at menarche for high and medium SES groups occurred relatively early, whereas that for the low SES observed last. In a US study found income status to be related to the timing of menarche over time when the proportion of girls experiencing early menarche ( $<11$ years old) increased over a 50 year time period only for those girls of a low socioeconomic position (39). In contrast, another study reported in the United Kingdom (UK) spanning an 85 year time period found a decline in the timing of menarche across all socioeconomic groups (40).

The results of studies on the relationship between menarche age and metabolic syndrome is not certain $(13,22,41-43)$. The most suggested explanation is that early menarche in adolescents and MetS in adulthood are consequences of childhood obesity (13).our study showed that early age at menarche is associated with a higher risk of MetS in women aged 35 to 70 years in HCS. Elsehely et al reported the statistically significant association between age at menarche in women with and without MetS (11.6 \pm 1.1 years vs.13.2 \pm 1.8 years respectively, $\mathrm{P}<0.001)(14)$. Hyse et al demonstrated in women with an age at menarche below 12.5 years Odds ratio of 1.49 (Cl: 1.22-1.82) for the MetS (43).Feng et al in Chinese women reported age at menarche was inversely associated with the total number of MetS components (41).

Lee et al suggested the risk of MetS was significantly increased at $\leq 12$ years of age at menarche $(\mathrm{OR}=1.91, \mathrm{P}<0$ 05) $(1)$. Gloeck et al showed menarche age had a curvilinear ('U' shaped) relationship with MetS in adulthood. Late menarche and early menarche are risk factors for MetS, and cardiometabolic abnormalities in adults (44). Cho et al showed no association between age at menarche and MetS (22). Also Hwang et al reported early or late menarche was not associated with an increased risk of MetS in premenopausal Korean women (2).

Janghorbani et al. showed that there is a strongly association between younger age at menarche and an increased risk of T2D (45). The results of a study in Brazil showed a relationship between menarche age (less than 11 years) and a high risk of diabetes (46). Iwase $\mathrm{M}$ et al in a retrospective cohort study on the relationship between menarche age and obesity and glycemic control in T2D showed that there was an inverse relationship between age at menarche and BMI and abdominal circumference (47). Yang et al. reported that menarche age has a linear and inverse relationship with the incidence of diabetes for each year of delay (48).Another study by Qiu et al demonstrated that no significant association between menarche age and diabetes. But there was a significant relationship between late menarche age (18 years) and lower CVD risk (10).

Lee et al reported Women with age at menarche $\leq 12$ years had significantly higher levels of triglycerides $(\beta$ coefficient $=37.83, P=0.02)$. Beside that hypertriglyceridemia was significantly increased at early menarche with 1.99 (95\% Cl: 1.16-3.41, P < 0 01) (1). Hozoori et al suggested Body mass index, waist circumference (WC), and Weight were inversely and significantly associated with the age of menarche $(P<0.01)(49)$. Also Wronka was showed a significant relationship between weight and BMI with the age at menarche (50)

\section{Strengths and Limitations of the Study:}

A major strength of the present study is the large study population. Another advantage was the use of high-reliability cohort study data.A limitation of our study is not having the actual menarche dates of the girls. However previous studies showed that self-reporting for menarche dates is reliable.

\section{Conclusion}

The present study showed the association between age at menarche and MetS in a large representative population.

\section{Declarations}

\section{Ethics approval and consent to participate:}

The study was confirmed by the ethics committee of Ahvaz Jundishapur University of Medical Sciences with a code of ethics IR.AJUMS.REC.1398.272. This study was conducted according to the Helsinki Declaration. On the day of the referral, we informed participants about all procedures involved, clearly. Informed written consent was obtained from the participants on the day of registration and notified participants can withdraw from the study whenever they wish.

Consent for Publication: Not applicable

Competing interests: None to declare

Author details: 
${ }^{1}$ Alimentary Tract Research Center, Clinical Sciences Research Institute, Department of Biostatistics and Epidemiology, School of Public Health, Ahvaz Jundishapur University of Medical Sciences, Ahvaz, Iran. ${ }^{2}$ Hearing Research Center, Clinical Sciences Research Institute, Department of Otolaryngology, Head and Neck Surgery, Ahvaz Jundishapur University of Medical Sciences, Ahvaz, Iran. ${ }^{3}$ Hearing Research Center, Clinical Sciences Research Institute, Department of Biostatistics and Epidemiology, School of Public Health, Ahvaz Jundishapur University of Medical Sciences, Ahvaz, Iran. ${ }^{4}$ Clinical Sciences Research Institute, Department of Biostatistics and Epidemiology, School of Public Health, Ahvaz Jundishapur University of Medical Sciences, Ahvaz, Iran. ${ }^{5}$ Alimentary Tract Research Center, Department of Internal Medicine, School of Medicine, Ahvaz Jundishapur University of Medical Sciences, Ahvaz, Iran. ${ }^{6}$ Clinical Sciences Research Institute, Ahvaz Jundishapur University of Medical Sciences, Ahvaz, Iran. ${ }^{7}$ Department of Biostatistics and Epidemiology, School of Public Health, Ahvaz Jundishapur University of Medical Sciences, Ahvaz, Iran. ${ }^{8}$ Fertility, Infertility and Perinatology Research Center, Department of Obstetrics and Gynecology, School of Medicine, Ahvaz Jundishapur University of Medical Sciences, Ahvaz, Iran.

\section{Availability of data and materials:}

The datasets used and analyzed during the current study are available from the corresponding author on reasonable request.

\section{Funding:}

The Iranian Ministry of Health and Medical Education has contributed to the funding used in the PERSIAN Cohort through Grant number $700 / 534$ and the Vice-Chancellor for Research at Ahvaz Jundishapur University of Medical Sciences grant number HCS-9809.

\section{Authors' contributions:}

NN and BCh were principal investigators of the study and drafted the manuscript. SJH, NS and ASh were advisors of the study. ASM, ZR and SS performed the statistical analysis. JK had role in data gathering. All authors contributed to the design and data analysis and assisted in the preparation of the final version of the manuscript. All authors read and approved the final version of the manuscript.

\section{Acknowledgments}

This project was approved by the ethics committee of Ahvaz Jundishapur University of Medical Sciences (Ethical code: IR.AJUMS.REC.1398.272).

\section{References}

1. Lee H-S, Leem S, Oh B, Park T. Effect of Interaction between Early Menarche and Genetic Polymorphisms on Triglyceride. Oxidative Medicine and Cellular Longevity. 2019; 2019:9148920.

2. Hwang Y-S, Park E-J, Choi J-G, Kim H-E, Park S-G, Yoo S-M. Relationship between Age at Menarche and Metabolic Syndrome in Premenopausal Women: Korea National Health and Nutrition Examination Survey 2013-2014. Korean J Fam Med. 2018; 39(5):300-6.

3. Castilho SD, Pinheiro CD, Bento CA, Barros-Filho Ade A, Cocetti M. [Secular trends in age at menarche in relation to body mass index]. Arquivos brasileiros de endocrinologia e metabologia. 2012; 56(3):195-200.

4. Ibitoye M, Choi C, Tai H, Lee G, Sommer M. Early menarche: A systematic review of its effect on sexual and reproductive health in low- and middle-income countries. PLoS One. 2017; 12(6):e0178884.

5. Charalampopoulos D, McLoughlin A, Elks CE, Ong KK. Age at menarche and risks of all-cause and cardiovascular death: a systematic review and metaanalysis. American journal of epidemiology. 2014; 180(1):29-40.

6. Jahanfar S, Lye M-S, Krishnarajah IS. Genetic and environmental effects on age at menarche, and its relationship with reproductive health in twins. Indian J Hum Genet. 2013;19(2):245-50.

7. Xing C, Huang Z, Li J, Li M, Xu L, Tao J, et al. Interactions of physical activity and body mass index with age at menarche: A school-based sample of Chinese female adolescents. European journal of obstetrics, gynecology, and reproductive biology. 2017;218:68-72.

8. Cassidy-Bushrow AE, Peters RM, Burmeister C, Bielak LF, Johnson DA. Neighborhood-Level Poverty at Menarche and Prepregnancy Obesity in AfricanAmerican Women. Journal of pregnancy. 2016;2016:4769121.

9. Bratke H, Bruserud IS, Brannsether B, Aßmus J, Bjerknes R, Roelants $M$, et al. Timing of menarche in Norwegian girls: associations with body mass index, waist circumference and skinfold thickness. BMC pediatrics. 2017;17(1):138.

10. Qiu C, Chen H, Wen J, Zhu P, Lin F, Huang B, et al. Associations between age at menarche and menopause with cardiovascular disease, diabetes, and osteoporosis in Chinese women. The Journal of clinical endocrinology and metabolism. 2013;98(4):1612-21.

11. Akter S, Jesmin S, Islam M, Sultana SN, Okazaki O, Hiroe M, et al. Association of age at menarche with metabolic syndrome and its components in rural Bangladeshi women. Nutrition \& Metabolism. 2012;9(1):99.

12. Stöckl D, Meisinger C, Peters A, Thorand B, Huth C, Heier M, et al. Age at menarche and its association with the metabolic syndrome and its components: results from the KORA F4 study. PLoS One. 2011;6(10):e26076.

13. Lim SW, Ahn JH, Lee JA, Kim DH, Seo JH, Lim JS. Early menarche is associated with metabolic syndrome and insulin resistance in premenopausal Korean women. European journal of pediatrics. 2016;175(1):97-104. 
14. Elsehely I, Abdel Hafez H, Ghonem M, Fathi A, Elzehery R. A Cutoff for Age at Menarche Predicting Metabolic Syndrome in Egyptian Overweight/Obese Premenopausal Women. Diabetes \& metabolism journal. 2017;41(2):146-9.

15. Gong TT, Wu QJ, Vogtmann E, Lin B, Wang YL. Age at menarche and risk of ovarian cancer: a meta-analysis of epidemiological studies. International journal of cancer. 2013;132(12):2894-900.

16. Lakshman R, Forouhi NG, Sharp SJ, Luben R, Bingham SA, Khaw KT, et al. Early age at menarche associated with cardiovascular disease and mortality. The Journal of clinical endocrinology and metabolism. 2009;94(12):4953-60.

17. Prentice P, Viner RM. Pubertal timing and adult obesity and cardiometabolic risk in women and men: a systematic review and meta-analysis. International journal of obesity (2005). 2013;37(8):1036-43.

18. Kivimäki M, Lawlor DA, Smith GD, Elovainio M, Jokela M, Keltikangas-Järvinen L, et al. Association of age at menarche with cardiovascular risk factors, vascular structure, and function in adulthood: the Cardiovascular Risk in Young Finns study. The American journal of clinical nutrition. 2008;87(6):187682.

19. Ley SH, Li Y, Tobias DK, Manson JE, Rosner B, Hu FB, et al. Duration of Reproductive Life Span, Age at Menarche, and Age at Menopause Are Associated With Risk of Cardiovascular Disease in Women. Journal of the American Heart Association. 2017;6(11).

20. Wang L, Yan B, Shi X, Song H, Su W, Huang B, et al. Age at menarche and risk of gestational diabetes mellitus: a population-based study in Xiamen, China. BMC pregnancy and childbirth. 2019;19(1):138.

21. Yang L, Li L, Millwood IY, Peters SAE, Chen Y, Guo Y, et al. Age at menarche and risk of major cardiovascular diseases: Evidence of birth cohort effects from a prospective study of 300,000 Chinese women. International Journal of Cardiology. 2017;227:497-502.

22. Cho YJ, Lee GH. Association of age at menarche with metabolic syndrome and components of metabolic syndrome in premenopausal women, Korea National Health and nutrition examination survey VI. International Journal of Diabetes in Developing Countries. 2016;36(3):345-51.

23. Farahmand M, Ramezani Tehrani F, Bahri Khomami M, Noroozzadeh M, Azizi F. Surgical menopause versus natural menopause and cardio-metabolic disturbances: A 12-year population-based cohort study. Journal of endocrinological investigation. 2015;38(7):761-7.

24. Dalvand S, Niksima SH, Meshkani R, Ghanei Gheshlagh R, Sadegh-Nejadi S, Kooti W, et al. Prevalence of Metabolic Syndrome among Iranian Population: A Systematic Review and Meta-analysis. Iranian journal of public health. 2017;46(4):456-67.

25. Cheraghian B, Hashemi SJ, Hosseini SA, Poustchi H, Rahimi Z, Sarvandian S, et al. Cohort profile: The Hoveyzeh Cohort Study (HCS): A prospective population-based study on non-communicable diseases in an Arab community of Southwest Iran. Medical journal of the Islamic Republic of Iran. 2020;34:141.

26. FARAHMAND M, RAMEZANI TEHRANI F, BEHBOUDI GANDEVANI S, AZIZI F. Is There any Association between Age at Menarche and Risk of Metabolic Syndrome? The Tehran Lipid \& Glucose Study. ARCHIVES OF IRANIAN MEDICINE. 2019;22(9):-

27. Poustchi H, Eghtesad S, Kamangar F, Etemadi A, Keshtkar AA, Hekmatdoost A, et al. Prospective Epidemiological Research Studies in Iran (the PERSIAN Cohort Study): Rationale, Objectives, and Design. American journal of epidemiology. 2018;187(4):647-55.

28. Royer M, Castelo-Branco C, Blümel JE, Chedraui PA, Danckers L, Bencosme A, et al. The US National Cholesterol Education Programme Adult Treatment Panel III (NCEP ATP III): prevalence of the metabolic syndrome in postmenopausal Latin American women. Climacteric. 2007;10(2):164-70.

29. Bahrami N, Soleimani MA, Chan YH, Ghojazadeh M, Mirmiran P. Menarche age in Iran: A meta-analysis. Iran J Nurs Midwifery Res. 2014;19(5):444-50.

30. Cabrera SM, Bright GM, Frane JW, Blethen SL, Lee PA. Age of thelarche and menarche in contemporary US females: a cross-sectional analysis. Journal of pediatric endocrinology \& metabolism: JPEM. 2014;27(1-2):47-51.

31. Talma H, Schönbeck Y, Van Dommelen P, Bakker B, Buuren S, Hirasing R. Trends in Menarcheal Age between 1955 and 2009 in the Netherlands. PloS one. 2013;8:e60056.

32. Biradar A, Yaliwal R, Kori S, Sangolli LS, Shirgur SS, Mathapati SS. A prospective cross-sectional study to assess the association of age of menarche with body mass index in adolescent girls of urban and rural schools of vijayapura, north karnataka. Journal of Krishna Institute of Medical Sciences University. 2020;9:72-80.

33. Khatoon T, Verma AK, Kumari R, Rupani R, Singh M, Rizvi A. Age at menarche and affecting bio-social factors among the girls of Lucknow, Uttar Pradesh. J Indian Acad Forensic Med. 2011;33(5):971-3.

34. Said-Mohamed R, Prioreschi A, Nyati LH, van Heerden A, Munthali RJ, Kahn K, et al. Rural-urban variations in age at menarche, adult height, leg-length and abdominal adiposity in black South African women in transitioning South Africa. Annals of Human Biology. 2018;45(2):123-32.

35. Ajong AB, Tankala NN, Yakum MN, Azenoi IS, Kenfack B. Knowledge of peri-menarcheal changes and a comparative analysis of the age at menarche among young adolescent school girls in urban and rural Cameroon. BMC Public Health. 2020;20(1):1661.

36. Ameade EP, Garti HA. Age at Menarche and Factors that Influence It: A Study among Female University Students in Tamale, Northern Ghana. PLoS One. 2016;11(5):e0155310.

37. Marván M, Castillo-López R, Del Callejo D, Canal-Martínez M, Nuñez-de la Mora A. Secular trends in age at menarche in 20th century Mexico: Differences by ethnicity, area of residency, and socioeconomic status. American Journal of Human Biology. 2020;32.

38. Latha Ramalingam AV. A cross-sectional study on the age of onset of menarche in females among

39. rural and urban areas of Kanchipuram district in the past 5 years,

40. since 2014. National Journal of Physiology, Pharmacy and Pharmacology. 2020;10(01):94-8.

41. Krieger N, Kiang MV, Kosheleva A, Waterman PD, Chen JT, Beckfield J. Age at menarche: 50-year socioeconomic trends among US-born black and white women. American journal of public health. 2015;105(2):388-97. 
42. Morris DH, Jones ME, Schoemaker MJ, Ashworth A, Swerdlow AJ. Secular trends in age at menarche in women in the UK born 1908-93: results from the Breakthrough Generations Study. Paediatric and perinatal epidemiology. 2011;25(4):394-400.

43. Feng $Y$, Hong X, Wilker E, Li Z, Zhang W, Jin D, et al. Effects of age at menarche, reproductive years, and menopause on metabolic risk factors for cardiovascular diseases. Atherosclerosis. 2008;196(2):590-7.

44. Frontini MG, Srinivasan SR, Berenson GS. Longitudinal changes in risk variables underlying metabolic Syndrome $X$ from childhood to young adulthood in female subjects with a history of early menarche: the Bogalusa Heart Study. International journal of obesity and related metabolic disorders: journal of the International Association for the Study of Obesity. 2003;27(11):1398-404.

45. Heys M, Schooling CM, Jiang C, Cowling BJ, Lao X, Zhang W, et al. Age of menarche and the metabolic syndrome in China. Epidemiology (Cambridge, Mass). 2007;18(6):740-6.

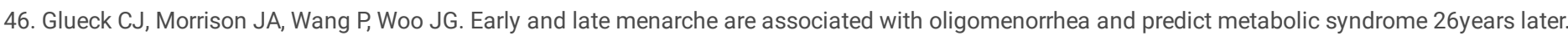
Metabolism. 2013;62(11):1597-606.

47. Janghorbani M, Mansourian M, Hosseini E. Systematic review and meta-analysis of age at menarche and risk of type 2 diabetes. Acta diabetologica. 2014;51(4):519-28

48. Mueller NT, Duncan BB, Barreto SM, Chor D, Bessel M, Aquino EM, et al. Earlier age at menarche is associated with higher diabetes risk and cardiometabolic disease risk factors in Brazilian adults: Brazilian Longitudinal Study of Adult Health (ELSA-Brasil). Cardiovascular diabetology. 2014;13:22.

49. Sumi A, Iwase M, Nakamura U, Fujii H, Ohkuma T, Ide H, et al. Impact of age at menarche on obesity and glycemic control in Japanese patients with type 2 diabetes: Fukuoka Diabetes Registry. Journal of diabetes investigation. 2018;9(5):1216-23.

50. Yang L, Li L, Peters SAE, Clarke R, Guo Y, Chen Y, et al. Age at Menarche and Incidence of Diabetes: A Prospective Study of 300,000 Women in China. American journal of epidemiology. 2018;187(2):190-8.

51. Hozoori M, Moradi F, Hosseini-zade Z, Kazemian M, Arsang-jang S. Age at Menarche and its Relationship to Anthropometric Indices in Adolescent Girls. International Journal of Pediatrics. 2017;5(7):5255-62.

52. Wronka I. Association between BMI and age at menarche in girls from different socio-economic groups. Anthropologischer Anzeiger; Bericht uber die biologisch-anthropologische Literatur. 2010;68(1):43-52. 